\title{
US Healthcare - We Must do Better!
}

David S*

Department of Clinical Medicine, New York University, USA

The BMJ recently reported that medical errors are the third leading cause of U.S. hospital mortality [1]. Impossible? Having practiced critical care in a large university hospital for 16 years, I can answer- not only is this possible, the majority of errors are unrecognized, unreported or not catastrophic. Though study design and third-party "coverage" of its results were condemned by many physicians, these reactions were either uninformed or conscious attempts to mask reality. Though not a RCT, the message it sends is quite clear: widespread, systematic changes are needed to rehabilitate the previous first-rate reputation of the US medical establishment.

Sicker patients increasingly occupy hospital beds; to a large extent, inpatient medicine is evolving into "critical care" [2]. Medical education, healthcare delivery, and the goals of our "leaders" have failed to meet this challenge. Rather, despite strong selection pressures, US healthcare delivery continues to diverge from a system concordant with demands.

Ideal treatment of a patient, especially an inpatient, relies on meticulous, hands-on, flexible and thoughtful care. Despite tremendous advances in medical technology and colossal growth of Information Technology, affording young doctors immediate access to oodles of information/data, these same providers, on average, are ill equipped to manage our current cohort of hospitalized patients.

Contributors to the current state are myriad and pervasive. From changes in medical school curricula, to work hour restrictions limiting care continuity, to acceptance, or even encouragement, of the "adequate" physician- the system is rapidly devolving!

The system may collapse at the bedside but it is the same "system" responsible for shaping our medical schools, the doctors they produce and the hospitals where they practice. On paper, recent medical school applicants are intelligent, well rounded and accomplished. Despite this, the art of relevant history taking and performing a skilled physical exam along with a thoughtful assessment of a patient's problems has nearly vanished! Refining the initial impression, much of which is done by talking to, and "laying hands" on, the patient, has become far less common.

Regulations, checklists, huddles (all potentially beneficial) arise to provide some framework to a chaotic system. Limitations on trainee working hours combined with the time-consuming and laborious need to ensure an accurate, complete (redundant) EMR leaves less time to spend with the patient. The relative disappearance of the physician at the bedside is evident to patients and families, contributes significantly to dissatisfaction, and, undoubtedly, is a prime cause of medical errors [3].

I understand the picture I've painted isn't pretty. I'm unhappy with the current state of U.S. medicine, but I am not nihilistic. We have taken our eyes off the ball. I remain hopeful that reemphasis on the doctor-patient relationship as a core objective will lead to a productive, vicious cycle, restoring respect and autonomy of physicians, bringing "the best and the brightest" back to medicine. With appreciation and time, new physicians will be energized, rededicating themselves to study the "basics", learning to combine "ancient" wisdom with modern advances. Doctors will spend more time with patients; errors will decrease. Quality will recover, care will be more efficient and satisfaction of all will improve.

\section{References}

1. Makary MA, Daniel M (2016) Medical error-the third leading casue of death in the US. BMJ 353: i2139.

2. American Hospital Association (2012) Are medicare patients getting sicker? TRENDWATCH.

3. Block L, Habicht R, Wu AW, Desai SV, Wang K, et al. (2013) In the wake of the 2003 and 2011 duty hours regulations, how do Internal medicine interns spend their time. J Intern Med 28: 1042-1047.
*Corresponding author: David Schwart, Intensivist Associate Professor of Clinical Medicine, New York University, 66 Edward St, Demarest, NJ 07627, USA, Tel: 2017840696; E-mail: davidschwartz1985@aol.com

Received July 09, 2016; Accepted August 30, 2016; Published September 07 2016

Citation: David S (2016) US Healthcare - We Must do Better! Health Care Curren Reviews 4: 174 . DOI: 10.4172/2375-4273.1000174

Copyright: (C) 2016 David S. This is an open-access article distributed under the terms of the Creative Commons Attribution License, which permits unrestricted use, distribution, and reproduction in any medium, provided the original author and source are credited. 